\title{
The State of Job Burnout Amongst New Zealand Managers: Implications for Employment Relations
}

\author{
JARROD HAAR*
}

\begin{abstract}
Job burnout is a perennial issue for workers, but the most popular way to measure it has become widely critiqued. We utilise the newest burnout construct - the Burnout Assessment Tool (BAT) - and provide New Zealand's first data on managers. Importantly, the BAT provides a cut-off score representing severe burnout (being burnt-out). Using data from $840 \mathrm{New}$ Zealand managers, support for the BAT construct is found, and 11.3 per cent of managers meeting the burnt-out threshold. Statistical analysis towards burnt-out status shows significant differences across managerial position, firm size, and workforce education, although no significant differences by sector. In light of growing attention to burnout, we discuss the implications for employment relations around burnout and the importance for firms to address this growing issue.
\end{abstract}

Keywords: burnout assessment tool; managers; New Zealand; position; firm factors.

\section{Introduction}

Schaufeli et al. (2019) define job burnout as "a work-related state of exhaustion that occurs among employees, which is characterised by extreme tiredness, reduced ability to regulate cognitive and emotional processes, and mental distancing" (p. 29). Job burnout is a critical issue for workers due to links with poor mental health (Schaufeli et al., 2019; Sakakibara et al., 2020; Schaufeli et al., 2020a, 2020b) and work outcomes, with meta-analyses highlighting many critical consequences. For example, Koutsimani et al.,'s (2019) meta-analysis found that burnout was positively related to anxiety and depression. Towards work outcomes, Swider and Zimmerman's (2010) meta-analysis found job burnout was linked positively to absenteeism and turnover, and negatively with job performance. The meta-analysis by Alarcon (2011) found negative links to job attitudes of job satisfaction and organisational commitment. Hence, we know that exploring burnout is an important issue.

While job burnout is critical to all workers, the present study focuses on managers. Haar et al., (2018) stated that "managers face unique and complex difficulties" (p. 2659), which makes exploring the burnout experiences of managers vital. Indeed, Roche and Haar noted that the quest for high quality leaders means that wellbeing is becoming more critical. However, the focus on leaders' job burnout has not received strong attention. We seek to explore the role that being a manager has on job burnout and whether characteristics of the firm and manager play a role. Notably, the burnout literature has taken a new turn recently. Schaufeli et al. (2019, 2020a, 2020b) has heavily critiqued the most popular burnout construct and offered an

\footnotetext{
* Professor, Department of Management, AUT University, Auckland, New Zealand 
alternative. Below, we briefly detail the critique and new approach and then test the new burnout construct on a large sample of New Zealand managers. Hence, this paper contributes to the burnout literature by providing new insights into New Zealand manager burnout and offers numerous implications for employment relations.

\section{Job Burnout Critique}

Job burnout was initially characterised by three dimensions: exhaustion, cynicism, and lack of professional efficacy (Maslach et al., 2001). The Maslach Burnout Inventory (MBI) has recently been strongly critiqued based on its dimensionality and cause and consequences (see Schaufeli et al., 2019, 2020a, 2020b). We briefly highlight the MBI critique due to it being the dominant construct in the burnout literature. Technical problems include extreme wording of some items, leaving very skewed respondent answers (Wheeler et al., 2011) and original anchors varying widely. The lowest score is "a few times a year or less" (score 1), and the next lowest being "once a month or less". This variation is problematic because the time distance between responses is not equivalent (Schaufeli et al., 2020a).

Another technical problem is around professional efficacy, in case of which the lower score indicates high burnout. Analyses have shown this dimension behaves differently from the rest of the burnout dimensions (Schaufeli et al., 2019, 2020a). Indeed, the correlation between emotional exhaustion and cynicism dimensions and the positive worded dimension of professional efficacy is lower than the correlation between the two detrimental dimensions (e.g., Schaufeli \& Van Dierendonck, 2000; Worley et al., 2008). A severe practical problem with the MBI measure is that there is no single burnout score but three separate scores for each dimension. Indeed, Maslach et al., (2017) state that the MBI constructs "should not be combined to form a single "burnout" score" (p. 44). Therefore, this aspect makes the MBI an investigative tool for burnout and not a diagnostic tool (Schaufeli et al., 2020a). Still, the implication that burnout is a 'syndrome' suggests that three different dimensions of burnout are interrelated. However, a second-order factor model, in which all three dimensions load on one common, underlying factor, shows that it does not fit with the data (Van Heule et al., 2012).

Finally, the MBI was not based on any theoretical notion (Schaufeli et al., 2019). Indeed, initial factor analysis included a fourth dimension, but this became neglected. Schaufeli et al. (2020a) argue that this inductive approach to burnout means some unpredictability around the burnout dimensions, which ultimately reflect poorly on the MBI. For instance, Deligkaris et al., (2014) argue that reduced cognitive functioning is established with burnout. Thus, burnout should cause issues with attention, working memory and concentration impairment. However, the MBI does not include a cognitive dimension. Similarly, the doubts around the inclusion of the personal accomplishment as a burnout dimension have been highlighted (Schaufeli \& Taris, 2005). They suggest that it may better be interpreted as a cause or consequence of burnout rather than a core dimension. A new construct was developed in response to these issues: The Burnout Assessment Tool or BAT (Schaufeli et al., 2019, 2020a).

\section{The Burnout Assessment Tool (BAT)}

From the above critique, a new approach to measure burnout was developed (BAT). It consists of four primary and three secondary dimensions. It can be used as a screening instrument to identify employees at risk for burning out, and as a diagnostic tool for assessing burnt-out employees. Schaufeli (2018) argued that the MBI has become insufficient to cope with the 
advancement and the measurement of burnout, especially in applied knowledge on epidemiology, assessment, prevention, treatment, and return to work. The BAT was developed through 49 in-depth, semi-structured interviews, including general practitioners, psychologists, and occupational physicians, who assess individuals before re-entry to the workplace, hence at the burnout process (De Witte \& Desart, 2019). These interviews focused on the symptomology of burnout and the work, interpersonal, and personality-related dynamic of burnout and its causes (De Witte \& Desart, 2019). The findings allowed to cluster identified symptoms of burnout into dimensions using the conceptual framework of burnout (Schaufeli \& Taris, 2005). Findings also provided insights into the causes and consequences of work-related burnout.

\section{BAT Dimensions}

BAT has four key dimensions: (1) exhaustion, (2) mental distance, (3) emotional impairment, and (4) cognitive impairment. These dimensions are combined into primary dimension due to theoretical understanding (Schaufeli \& Taris, 2005). The primary dimensions of BAT refer to the inability (exhaustion, impaired emotional and cognitive control) or the unwillingness (mental distance) to exert effort at work. Exhaustion is the most apparent symptom of burnout and is characterised by "tense emotional reactions and feeling overwhelmed by one's emotions. Specific symptoms include feeling frustrated and angry at work, irritability, overreacting, feeling upset or sad without knowing why, and feeling unable to control one's emotions at work" (Schaufeli et al., 2019, p. 27). Exhaustion is a necessary but not a sufficient dimension of burnout alone (De Witte \& Desart, 2019).

Mental distance refers to "psychologically distancing oneself from the work" and "...is indicated by a strong reluctance or aversion to work" (Schaufeli et al., 2019, p. 28). Thus, mental distance refers to a withdrawing mentally from work, with such employees being psychologically uninterested in the job or employment. This means that they avoid contact with others (e.g., customers, co-workers) and have an indifferent and cynical attitude (Schaufeli et al., 2019). With mental distance, employees' function is on autopilot and have little interest in their job. Emotional impairment "manifests itself in intense emotional reactions and feeling overwhelmed by one's emotions. Specific symptoms include feeling frustrated and angry at work, irritability, overreacting, feeling upset or sad without knowing why, and feeling unable to control one's emotions at work" (Schaufeli et al., 2019, p. 27). It is referred to as the reduced functional capacity to adequately regulate emotional processes (De Witte \& Desart, 2019), leading to overreaction and the inability to control emotions at work.

Finally, Schaufeli et al. (2019) define cognitive impairment as "memory problems, attention and concentration deficits and poor cognitive performance" (p. 27). This impairment includes symptoms of difficulty thinking clearly, inability to learn new things at work, being absentminded, and having deficiencies in attention, concentration, and memory (Schaufeli et al., 2019). Cognitive impairment refers to the reduced ability to process or regulate cognitive functions relating to memory and attention. It leads to forgetful or absentminded behaviours, poor memory, and lack of concentration at work (De Witte \& Desart, 2019).

\section{BAT Supporting Evidence}

Evidence has shown that these four dimensions relate to a higher-order construct of burnout (see Schaufeli et al., 2020a, 2020b; Spagnoli et al., 2021; Sakakibara et al., 2020; Hadibarjramovic et al., 2020). Notably, the BAT construct has a threshold cut-off representing very high burnout (Schaufeli et al., 2020a, 2020b) or burnt-out. Schaufeli et al. (2020a) refer 
to those in the highest-scoring zone (red zone) as suffering severe burnout, in effect, being burnt-out. Individuals in the next band or zone (orange zone) are categorised as being at high risk of burning out. The remainder (and majority) are seen as being in a green zone - low risk of burnout. Here, we refer to being burnt out as the critical point where burnout has become so intense that it severely affects workers. Their data reported burnt-out rates of five per cent in the Netherlands and eight per cent in Belgium (Schaufeli et al., 2020a).

Studies have supported the BAT construct and shown significant consequences, including higher turnover intentions, lower work engagement (Sakakibara et al., 2020), and increased psychological and psychosomatic complaints (Nellestijn, 2019; Spagnoli et al., 2021; Sakakibara et al., 2020). Similarly, several causes are positively related to burnout, including various job demands and workaholism (Sakakibara et al., 2020), job insecurity (Nellestijn, 2019), and perfectionism concerns (Spangnoli et al., 2021). Recent work (Otto et al., 2021a, 2021b) has shown proactive burnout prevention, self-efficacy, and optimism were negatively related to burnout. Finally, De Beer et al. (2020) investigated the measurement invariance of the BAT using seven different cross-national representative samples, including the Netherlands, Belgium, Austria, Ireland, Finland, and Japan. This study found invariant for BAT across all samples. However, New Zealand remains currently untested. Given the consistent evidence around BAT in European samples and Japan, we argue that the BAT factor structure will hold in New Zealand. This leads to the initial hypothesis.

Hypothesis 1. The BAT dimensions around (a) exhaustion, (b) mental distance, (c) impaired emotional control, and (d) impaired cognitive control will similarly hold with a New Zealand sample.

\section{Hypotheses}

While the paper aims to establish the BAT construct in New Zealand and establish a benchmark for burnt-out, we also test four hypotheses, including one on the individual and three at firmlevel factors. We see these as largely exploratory because our focus here on burnout and, specifically, being burnt-out amongst managers allows a greater focus on firm characteristics, which are largely missing from the field. We expect a positive relationship between managerial position and job burnout. Schieman and Reid (2009) argued and found that managers in senior management positions were more likely to be stressed. A senior managerial position reflects the complexities of their job (Haar et al., 2018), with Roche et al. (2018) noting that managing turbulent environments ultimately takes a toll on leaders' wellbeing. However, Warr (2005) argued that senior managers have greater freedom and decision-making latitude, and Roche and Haar (2010) did find benefits from senior management positions over junior managers. Ultimately, we expect being burnt-out to differ by managerial position. We posit the following.

\section{Hypothesis 2: Being burnt-out will differ by managerial position.}

We also test three firm factors due to their potential influence on burnout. Robertson and Seneviratne (1995) conducted a meta-analysis and found that the public sector setting can be more favourable than the private sector, albeit on some factors only. Kuoppala et al. (2008) indicate this likely includes wellbeing, suggesting similar effects towards job burnout. Ultimately, we expect differences in job pressures and workloads to differ by sector, and burntout levels to differ. Next, we test firm size. This is because larger-sized firms are likely to have more significant resources and might provide better human resource practices (see Haar et al., 
2021). However, larger-sized firms are also likely to face greater competition (Haar et al., 2021), thus creating tremendous pressure on managers. Again, we expect managers are reporting being burnt-out to differ across different sized firms. Finally, we test the role of workforce education composition towards manager burnout. We suggest higher educated workforces provide a more complex managerial role and are likely to be incredibly challenging, leading to greater burnt-out levels. Overall, we posit the following for the firm-level factors.

Hypothesis 3: Towards being burnt-out, sector differences will exist.

Hypothesis 4: Towards being burnt-out, firm size differences will exist.

Hypothesis 5: Towards being burnt-out, differences will exist by workforce education.

\section{Methods}

\section{Participants and Sample}

Data for the present study were collected from a Qualtrics survey panel. Qualtrics has an extensive database of many thousands of New Zealanders, including managers. Due to the focus on this sub-group, only managers were targeted by Qualtrics (for more details on Qualtrics, see Haar et al., 2021). Qualtrics invites potential respondents to complete the online survey and have their responses captured. The exact number approached and recruited is not available to the researcher. However, Qualtrics can recruit managers across a range of sectors, industries, and the geographical spread of New Zealand. In total, 840 manager responses were obtained early in 2020. The Qualtrics platform ensures quality respondents by removing too fast or slow respondents and ensuring only one response.

This panel approach had yielded positive samples (e.g., Ghafoor \& Haar, 2019). A metaanalytic comparison by Walter et al. (2019) found no significant difference between panel sourced data and conventional sourced data (e.g., mail surveys). We followed recommendations by Podsakoff et al. (2003) around potential common method bias (CMB) and separated our study factors by unrelated questions. This separation approach included the burnout items with other firm related questions interspaced. Respondents were senior executives (27.8 per cent), middle-level managers (50.0 per cent) and low-level managers (22.2 per cent), with average tenure in their current job being 7.99 years $(\mathrm{SD}=6.3)$. By firm size, the majority were large-sized ( 251 or more employees) at 47.5 per cent, followed by medium-sized (51-250 employees) at 32.3 per cent.

\section{Measures}

Job Burnout was measured using the 23 -items of the BAT scale, coded $1=$ never to $5=$ always. This coding follows the BAT manual (Schaufeli et al., 2020a) although some studies (e.g., Sakakibara et al., 2020) have used a different range. Given that this is the first New Zealand study, we call the instrument BAT-NZ. The items measure the four core symptoms of burnout: exhaustion (8-items, sample "At work, I feel physically exhausted" $\alpha=0.92$ ), mental distance (5-items, sample "I feel a strong aversion towards my job", $\alpha=0.89$ ), emotional impairment (5items, sample "At work I may overreact unintentionally", $\alpha=0.93$ ), and cognitive impairment (5-items, sample "At work I struggle to think clearly", $\alpha=0.92$ ). 
Fundamentally, exhaustion and mental distance are based initially on the MBI, with Roche and Haar (2013) suggesting that these are the core dimension of burnout. However, the new BAT construct created new items for these dimensions based on 49 interviews with general practitioners, occupational physicians, and psychologists who deal with burnout (Schaufeli et al., 2020a). Similarly, their interviews and subsequent survey development created new constructs around impairment (emotional and cognitive), new to the broad burnout literature. Impairment has been identified in the literature as occurring with burnout (see Deligkaris et al., 2014), but was missing from the MBI. In their study of 1500 employees from Flanders and 1500 employees from the Netherlands, Schaufeli et al. (2020a, 2020b) showed that the data fit the constructs best across the four dimensions. The analysis showed that the four dimensions are highly correlated $(.82<\mathrm{r}>.54)$, which makes combining the dimensions appropriate.

Confirmatory factor analysis (CFA) was conducted in AMOS (version 26) to confirm the BATNZ factor structure. Regarding goodness-of-fit indices, common guidelines in the literature were followed (e.g., Hu \& Bentler, 1998; Williams et al., 2009): (1) the comparative fit index (CFI $\geq .90$ ), (2) the Tucker-Lewis index (TLI $\geq .90$ ), (3) the root-mean-square error of approximation (RMSEA $\leq .08$ ), and (4) the standardised root mean residual (SRMR $\leq .10$ ). See Table 1. 
Table 1. Results of Confirmatory Factor Analysis BAT-NZ

\begin{tabular}{|c|c|c|c|c|c|c|c|c|c|c|}
\hline \multirow[b]{2}{*}{ Model } & \multicolumn{4}{|c|}{ Model Fit Indices } & \multicolumn{6}{|c|}{ Model Differences } \\
\hline & $\chi^{2}$ & Df & CFI & TLI & RMSEA & $\begin{array}{l}\text { SRMR } \\
\end{array}$ & $\chi^{2}$ & $\Delta \mathbf{d f}$ & $\mathbf{p}$ & Details \\
\hline Model 1 & 1209.8 & 224 & .93 & .93 & .07 & .05 & & & & \\
\hline Model 2 & 1717.0 & 227 & .90 & .89 & .09 & .06 & 507.2 & 3 & .001 & Model 1 to 2 \\
\hline Model 3 & 1666.4 & 227 & .90 & .89 & .09 & .06 & 456.6 & 3 & .001 & Model 1 to 3 \\
\hline Model 4 & 1716.1 & 227 & .90 & .89 & .09 & .06 & 506.3 & 3 & .001 & Model 1 to 4 \\
\hline
\end{tabular}

Model 1=Hypothesised 4-factor model of BAT: exhaustion, mental distance, emotional impairment, and cognitive impairment.

Model 2=Alternative 3-factor model: exhaustion and mental distance combined, emotional impairment, and cognitive impairment.

Model 3=Alternative 3-factor model: exhaustion, mental distance, emotional impairment and cognitive impairment combined.

Model 4=Alternative 3-factor model: exhaustion, mental distance and cognitive impairment combined, and emotional impairment. 
The model was an excellent fit to the data: $\chi^{2}(\mathrm{df})=1209.8(229), \mathrm{CFI}=.93$, RMSEA=.07, and $\mathrm{SRMR}=.05$. To confirm that the hypothesised construct structure was the most appropriate, alternative CFAs were tested. These models combined the various dimensions (e.g., exhaustion and mental distance, and emotional and cognitive impairment). See Table 1. A chi-squared difference test showed that these were all significantly a poor fit $(\mathrm{p}<.001)$ compared to the hypothesised model (Hair et al., 2010).

We next tested a higher-order model with the four dimensions loading onto a single factor (job burnout), and this demonstrated an excellent fit to the data: $\chi^{2}(\mathrm{df})=1319.2(229), \mathrm{CFI}=.93$, RMSEA=.08, and SRMR=.06. The combined construct follows the logic of Hadzibajramovic et al., (2020) and had excellent reliability $(\alpha=.96)$.

Burnt-Out was calculated using guidelines from Schaufeli et al. (2020a, 2020b). Using the burnout score above, we calculated being burnt out using the cut-off of $\geq 3.30$, which Schaufeli et al. (2020a) refer to as employees being in the red zone or those most likely suffering from serious burnout. Here, we refer to this as being burnt-out. We coded respondents 1 =burnt-out (job burnout Mean $\geq 3.30$ ), $0=$ job burnout Mean < 3.30). The calculation of the burnt-out scale threshold was done using Rasch analysis. Hadzibajramović et al. (2020) state that such analysis "belongs to the modern psychometric approaches or item response theory" (p. 3). Originally from education, it is widely used in the health sciences (see Christensen et al., 2013). Hadzibajramović et al. (2020) state that "the Rasch analysis allows a unified approach to measurement issues such as unidimensionality, appropriate category ordering of polytomous items, testing the invariance of items, and differential item functioning" (p. 3). As noted earlier, the categorisation of being burnt-out is new to any burnout construct and marks a new direction for the literature.

\section{Factors}

Managerial Position was measured by asking managers their position. Responses were coded $1=\mathrm{CEO} /$ highest ranked manager position, $2=$ executive/senior manager, $3=$ middle manager, and 4=low-level manager.

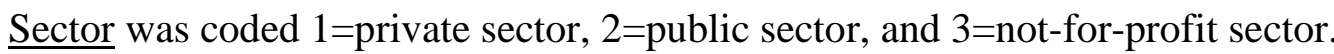

Firm Size was coded 1=micro-sized firm (1-10 employees), 2=small-sized firm (11-50 employees), $3=$ medium-sized firm (51-250 employees), and 4=large-sized firm (251+ employees).

Workforce Education was coded on the average workforce education: 1=high school qualification, $2=$ polytechnic qualification, $3=$ bachelor's degree, $4=$ postgraduate degree.

Analysis

Hypothesis 1 was tested above using CFA in AMOS (version 26). Hypotheses 2-5 were tested in SPSS (version 26) using ANOVA. Following Haar et al., (2014), we used the Fisher's Least Significant Difference and Student-Newman-Keuls tests for post-hoc analyses.

\section{Results}

The CFA in AMOS (Table 1) supports Hypothesis 1. Descriptive statistics for the study variables are shown in Table 2. 
Table 2. Correlations and Descriptive Statistics of Study Variables

\begin{tabular}{lllllllll}
\hline Variables & Mean & SD & 1 & 2 & 3 & 4 & 5 & 6 \\
\hline 1. Managerial Position & 2.63 & .91 & -- & & & & & \\
2. Sector & 1.3 & .57 & $.07 *$ & -- & & & & \\
3. Firm Size & 2.51 & 1.11 & $.33^{* *}$ & $.14 * *$ & -- & & & \\
4. Workforce Education & 2.40 & .99 & $-.08^{*}$ & $.18^{* *}$ & $.08^{*}$ & -- & & \\
5. Job Burnout & 2.37 & .76 & $.08^{*}$ & -.04 & $.19 * *$ & $.12 * *$ & -- & \\
6. Burnt-Out & .11 & .32 & -.04 & -.06 & $.10^{* *}$ & $.10^{* *}$ & $.67 * *$ & -- \\
\hline
\end{tabular}

$\mathrm{N}=840, * \mathrm{p}<.05, * * \mathrm{p}<.01$.

Table 2 shows that towards the overall job burnout construct, managerial position is positively related $(\mathrm{r}=.08, \mathrm{p}=.016)$, sector is not significantly correlated $(\mathrm{r}=-.04, \mathrm{p}=.213)$, firm size is positively correlated $(\mathrm{r}=.19, \mathrm{p}<.01)$, as is workforce education $(\mathrm{r}=.12, \mathrm{p}<.01)$. The analysis showed that 11.3 per cent of managers reported being burnt out.

With the dependent variable being the burnt-out rate, the ANOVA results are presented here with the various antecedents. First are differences by managerial position. ANOVA showed significant differences $(\mathrm{F}=3.411, \mathrm{p}=.017)$, with the significantly highest burnt-out rate from executives/senior managers at 17 per cent. This rate was significantly higher than middle-managers (10 per cent), which, in turn, was significantly higher than CEOs/highest ranked manager (eight per cent) and low-level manager (eight per cent). By firm size, ANOVA showed significant differences $(\mathrm{F}=8.579, \mathrm{p}<.001)$, with the significantly highest burnt-out rate from medium-sized firms (19 per cent). This rate was significantly higher than large-sized firms (11 per cent), small-sized firms (11 per cent) and microsized firms (four per cent). ANOVA showed no significant differences by sector $(\mathrm{F}=2.285, \mathrm{p}=.102)$. Finally, by workforce education, ANOVA showed significant differences $(\mathrm{F}=2.970, \mathrm{p}=.031)$, with the significantly highest burnt-out rate from managers with workforces holding mostly postgraduate qualifications (15 per cent), followed by workforces with mostly bachelor's degrees (13 per cent), which was significantly higher than workforces with mostly polytechnic qualifications (12 per cent). Managers controlling mostly high school qualified workforces reported the lowest burnout rates, significantly (six per cent). Overall, these findings support Hypotheses 2, 4, and 5.

\section{Discussion}

The present study sought to establish the BAT-NZ and, thus, extend the number of countries that have supported the new BAT construct. This adds to the work of De Beer et al. (2020), confirming BAT across seven samples (Netherlands, Belgium, Austria, Ireland, Finland, and Japan). While the present study is not a nationally representative sample due to its focus on managers, it does provide a much needed start to BAT-NZ, confirming the factor structure of BAT amongst managers. Importantly, this study found a burnt-out rate of 11.3 per cent, which is significantly higher than five per cent in the Netherlands and eight per cent in Belgium (Schaufeli et al., 2020a). This higher score might reflect the fact the present study is managers only. However, it also provides initial insights into burnout and severe burnout rates (being burnt-out) in the New Zealand workforce, with BAT data currently being missing from the literature. The data provides valuable insights, indicating that executives/senior 
managers bear the brunt of being burnt-out, supporting Schieman and Reid (2009), but CEOs were significantly lower, which supports arguments around greater freedom and latitude (Warr, 2005; Roche \& Haar, 2010).

Our analyses also showed that medium-sized firms had managers most likely to be burnt-out, which might reflect larger firms having more advanced HR practices (see Haar et al., 2021), and, thus, managers in these firms might be especially stretched. As with managerial position, this highlights the complexity of management and argues for greater delineation when assessing burnt-out rates as we did here (by position and firm size). Sector differences were not supported, but significant differences through managing more educated workforces were found towards burnt-out rates. These results were more linear and showed that, as the workforce becomes more educated, there are growing pressures on managers, leading to higher burnt-out rates. Overall, our analyses provide much needed insights into our understanding of experiencing burnt-out and where this occurs. This provides a much needed initial picture of being burnout in New Zealand. We next focus on the employment relations implications from these findings.

\section{Implications}

The evidence around burnout and being burnt-out has severe implications for human resource (HR) managers. Within their training and development, managers need to manage their workloads and ability to cope. We suggest that targeted training around the warning signs of burnout and becoming burnt-out are needed. This attention to workload also aligns with New Zealand legislation, especially around occupational health and safety, which has growing interest in occupations (see Lamm et al., 2017). The Health and Safety in Employment Amendment Act 2002 highlights that employers are responsible for workplace harm. Section 2(1) of the Act has greater clarification around harm, with the Amendment stating that harm "includes physical or mental harm caused by work-related stress" (p. 6). Given that the literature identifies various job demands factors and job insecurity as important burnout antecedents (Sakakibara et al., 2020; Nellestijn, 2019), these are issues that firms ultimately need to manage better or be liable for neglectful practice.

Similarly, managers may face high work demands and workload issues, perhaps exacerbated by connectivity, which means firms need to address and alleviate burnout as a symptom and, ultimately, the consequences of burnout. These consequences include job satisfaction (Schaufeli et al., 2020a), which is a crucial determinant of job performance (Judge et al., 2001). Similarly, evidence shows that burnout is negatively related to work engagement (Sakakibara et al., 2020), which also enjoys metaanalytic support towards job performance (Christian et al., 2011). The HR focus on workforce engagement is critically hampered if managers - especially those overseeing others - suffer from burnout or are burnt-out.

Beyond performance implications, workers with high burnout are more likely to consider quitting their job (Sakakibara et al., 2020), and this is especially problematic when applied to managers. Turnover is a business cost and negatively affects firm performance (Park \& Shaw, 2013), and the costs associated with turnover increase commensurate with worker pay (Cascio \& Boudreau, 2008). Hence, as an expensive human resource, managers become an additional high cost to a firm when they leave. Indeed, Rasmussen et al. (2014) highlighted the importance of managerial skills in deciphering the employee productivity 'conundrum'. Loss of institutional knowledge, positive relationships with subordinates, ease of replacement etc., all impact firm operations. This can have implications on HR issues around recruitment, selection, and retention. How easy is it to recruit for a role where a manager has just burnt-out? What steps are needed to help retain other managers working in the same climate? Recruiting more resilient managers might be an option, but this is likely focusing on the wrong factors. 
The meta-analysis by Aronsson et al. (2017) identified workloads as critical to burnout and job control, and autonomy as critical ways burnout can be managed and reduced. Ensuring managers have maximum autonomy and control is, thus, key. Finally, beyond managers' mental wellbeing (Nellestijn, 2019; Spagnoli et al., 2021; Sakakibara et al., 2020), there are additional wellbeing issues around sleep and eating behaviours (see Corr et al., 2017).

Implications also abound from the workforces being managed. The findings suggest that highly educated workforces provide additional challenges for managers; thus, employers need to be aware of this issue. While the gig economy has growing attention (see Sarina \& Riley, 2018), this would also fit within our burnt-out profile if such workforces were more educated. However, managing a gig workforce might have additional challenges, as workers choose to engage (or not) with work at their leisure. While not a focus of the present study, this is an avenue for future studies. Further, burnout might have implications for the quality of democratic engagement. Novitz (2019) states that employment conditions can limit the energy "for political activity and campaigning" (p. 8). In the context of managers, this would leave them with reduced potential to engage with owners. Being burntout also leaves them out of supporting their own subordinates' democratic engagement due to complications from being burnt-out. Thus, some managers will lose their democratic voice and support for such democracy, which might be necessary where pro-employee support is especially needed.

Finally, studies have shown the importance of identity for workers (see Smollan \& Pio, 2017). What are the implications of managers being burnt-out? The potential to be a 'badge of honour' might harken back to a golden age of capitalism (e.g., the 1980s). Still, it might also be a critical issue if being a manager is fundamentally tied to a worker's identity. What does this mean when a manager is seen as burnt-out? Is this a positive outcome or an indication of a deficit and weakness? Ultimately, we do not know. But this has rich research implications to understanding the psyche of managers. Indeed, given perfectionism is linked to burnout (Spangnoli et al., 2021), there might be perverse identity issues at play, driving a manager to become burnt-out instead of stepping away or taking sufficient break time. We encourage researchers to explore these myriad employment implications to enhance our understanding. One such example might be the role of the CEO or top management team and the influence they have over managers, especially their workload. Again, research opportunities abound.

\section{Conclusion}

In summary, the present study explored the new burnout measure (BAT-NZ) in the New Zealand setting, and suitable psychometric properties were supported. Notably, the ability to calculate a burntout score identified an 11.3 per cent score for this sample of New Zealand managers. While higher than other data (see Schaufeli et al., 2020a), that study focused on representative worker populations and not managers as in the present study. Perhaps the higher percentage burnt-out rate found here reflects this issue. Overall, the ANOVA showed several differences across managerial position, firm size, and workforce education, providing insights, which lead to managers reporting being burnt-out. Given the catastrophic consequences of burnout and being burnt-out, we encourage managers to focus not only on their subordinates' workload volumes and demands, but also on their own. Our findings provide a critical warning bell for managers' wellbeing. 


\section{References}

Alarcon, G. M. (2011). A meta-analysis of burnout with job demands, resources, and attitudes. Journal of Vocational Behavior, 79(2), 549-562.

Aronsson, G., Theorell, T., Grape, T., Hammarström, A., Hogstedt, C., Marteinsdottir, I., Skoog, In., Träskman-Bendz, L., \& Hall, C. (2017). A systematic review including meta-analysis of work environment and burnout symptoms. BMC Public Health, 17(1), 1-13. https://doi.org/10.1186/s12889-017-4153-7

Cascio, W., \& Boudreau, J. (2008). Investing in People. Upper Saddle Ridge.

Christian, M. S., Garza, A. S. \& Slaughter, J. E. (2011). Work engagement: A quantitative review and test of its relations with task and contextual performance. Personnel Psychology, 64(1), 89136.

Christensen, K. B., Kreiner, S., \& Mesbah, M. (2013). Rasch Models in Health. John Wiley \& Sons, Inc.

Corr, L., Dixon, J., \& Burgess, J. (2017). Early childhood education and care workers and wellbeing in a continuous caring regime. New Zealand Journal of Employment Relations, 42(3), 46-62.

De Beer, L. T., Schaufeli, W. B., De Witte, H., Hakanen, J. J., Shimazu, A., Glaser, J., ... \& Rudnev, M. (2020). Measurement invariance of the Burnout Assessment Tool (BAT) across seven cross-national representative samples. International Journal of Environmental Research and Public Health, 17(15), 5604. https://doi.org/10.3390/ijerph17155604

De Witte, H. \& Desart, S. (2019). Burnout 2.0 - A New Look at the Conceptualization of Burnout. In, Taris, T. W., Peeters, M. C. W., \& De Witte, H. (Eds), The Fun and Frustration of Modern Working Life: Contributions from an Occupational Health Psychology Perspective (pp. 140152). Pelckmans Pro: Kalmthout, Belgium.

Deligkaris, P., Panagopoulou, E., Montgomery, A. J., \& Masoura, E. (2014). Job burnout and cognitive functioning: A systematic review. Work \& Stress, 28(2), 107-123.

Ghafoor, A., \& Haar, J. (2019). Organisational-based self-esteem, meaningful work, and creativity behaviours: A moderated mediation model with supervisor support. New Zealand Journal of Employment Relations, 44(3), 11-31.

Haar, J., O'Kane, C., \& Daellenbach, U. (2021). High Performance Work Systems and Innovation in New Zealand SMEs: Testing Firm Size and Competitive Environment Effects. The International Journal of Human Resource Management, 1-29. https://doi.org/10.1080/09585192.2021.1894213

Haar, J. M., Roche, M., \& ten Brummelhuis, L. (2018). A daily diary study of work-life balance in managers: Utilising a daily process model. The International Journal of Human Resource Management, 29(18), 2659-2681. 
Haar, J. M., Russo, M., Sune, A. \& Ollier-Malaterre, A. (2014). Outcomes of work-life balance on job satisfaction, life satisfaction and mental health: A study across seven cultures. Journal of Vocational Behavior, 85(3), 361-373.

Hadzibajramović, E., Schaufeli, W., \& De Witte, H. (2020). A Rasch analysis of the Burnout $\begin{array}{llll}\text { Assessment } & \text { Tool } & \text { (BAT). Plos } & \text { One, 15(11), }\end{array}$ https://doi.org/10.1371/journal.pone.0242241

Hair, J. F., Black, W. C., Babin, B. J., \& Anderson, R. E. (2010). Multivariate Data Analysis (7th ed.). Pearson Prentice Hall.

Hu, L. T., \& Bentler, P. M. (1998). Fit indices in covariance structure modeling: Sensitivity to underparameterized model misspecification. Psychological Methods, 3(4), 424-453.

Judge, T. A., Thoresen, C. J., Bono, J. E., \& Patton, G. K. (2001). The job satisfaction-job performance relationship: A qualitative and quantitative review. Psychological Bulletin, 127, 376-407.

Koutsimani, P., Montgomery, A., \& Georganta, K. (2019). The relationship between burnout, depression, and anxiety: A systematic review and meta-analysis. Frontiers in Psychology, 10, 284. https://doi.org/10.3389/fpsyg.2019.00284

Kuoppala, J., Lamminpää, A., Liira, J., \& Vainio, H. (2008). Leadership, job well-being, and health effects-A systematic review and a meta-analysis. Journal of Occupational and Environmental Medicine, 50(8), 904-915.

Lamm, F., Moore, D., Nagar, S., Rasmussen, E., \& Sargeant, M. (2017). Under pressure: OHS of vulnerable workers in the construction industry. New Zealand Journal of Employment Relations, 42(2), 39-60.

Maslach, C., Jackson, S. E., Leiter, M. P., Schaufeli, W. B. \& Schwab, R. L (2017). Maslach Burnout Inventory Manual ( $4^{\text {th }}$ Edition). Mind Garden.

Maslach, C., Schaufeli, W. B., \& Leiter, M. (2001). Job Burnout. Annual Reviews Psychology, 52, 397-422.

Nellestijn, E. (2019). The Effect of Job Insecurity on the Well-Being of Employees: The Relationship between Job Insecurity, Burnout, and Work Engagement and the Moderating Role of Age and Openness to Experience [Unpublished master's thesis. Utrecht University.

Novitz, T. (2019). The perils of collective begging: The case for reforming collective labour law globally and locally too. New Zealand Journal of Employment Relations, 44(2), 3-19.

Otto, M. C., Van Ruysseveldt, J., Hoefsmit, N., \& Van Dam, K. (2021a). Examining the mediating role of resources in the temporal relationship between proactive burnout prevention and burnout. BMC Public Health, 21(1), 1-15.

Otto, M. C., Van Ruysseveldt, J., Hoefsmit, N., \& Van Dam, K. (2021b). Investigating the temporal relationship between proactive burnout prevention and burnout: A four-wave longitudinal study. Stress and Health. 1-12. https://doi.org/10.1002/smi.3037 
Park, T. Y., \& Shaw, J. D. (2013). Turnover rates and organisational performance: A metaanalysis. Journal of Applied Psychology, 98(2), 268-309.

Podsakoff, P. M., MacKenzie, S. B., Lee, J.-Y., \& Podsakoff, N. P. (2003). Common method biases in behavioral research: A critical review of the literature and recommended remedies. Journal of Applied Psychology, 88(5), 879-903.

Rasmussen, E., Fletcher, M., \& Hannam, B. (2014). The major parties: National's and Labour's employment relations policies. New Zealand Journal of Employment Relations, 39(1), 21-32.

Robertson, P. J., \& Seneviratne, S. J. (1995). Outcomes of planned organisational change in the public sector: A meta-analytic comparison to the private sector. Public Administration Review, 55(6), 547-558.

Roche, M., \& Haar, J. M. (2010). Work-family interface predicting needs satisfaction: The benefits for senior management. Journal of Social \& Behavioural Research in Business, 1(1), 12-23.

Roche, M., \& Haar, J. M. (2013). Leaders life aspirations and job burnout: A self-determination theory approach. Leadership \& Organization Development Journal, 34(6), 515-531.

Roche, M. A., Haar, J. M., \& Brougham, D. (2018). Māori leaders' well-being: A self-determination perspective. Leadership, 14(1), 25-39.

Sakakibara, K., Shimazu, A., Toyama, H., \& Schaufeli, W. B. (2020). Validation of the Japanese Version of the Burnout Assessment Tool. Frontiers in Psychology, 11, 1819.

Sarina, T., \& Riley, J. (2018). Re-crafting the enterprise for the gig-economy. New Zealand Journal of Employment Relations, 43(2), 27-35.

Schaufeli, W., De Witte, H., \& Desart, S. (2019). Manual Burnout Assessment Tool (BAT). KU Leuven.

Schaufeli, W.B. (2018). Burnout: Facts and Fiction, The Psychologist, 53(9), 10-20.

Schaufeli, W.B., De Witte, H. \& Desart, S. (2020a). Manual Burnout Assessment Tool (BAT) - Version 2.0. KU Leuven, Belgium: Unpublished internal report.

Schaufeli, W. B., Desart, S., \& De Witte, H. (2020b). Burnout Assessment Tool (BAT)Development, Validity, and Reliability. International Journal of Environmental Research and Public Health, 17(24), 9495. https://doi.org/10.3390/ijerph17249495

Schaufeli, W. B., \& Taris, T. W. (2005). The conceptualisation and measurement of burnout: Common ground and worlds apart. Work \& Stress, 19(3), 256-262.

Schaufeli, W. B., \& Van Dierendonck, D. (2000). Utrecht Burnout Scale (UBOS): Manual [Manual Utrecht Burnout Scale]. Swets \& Zeitlinger.

Schieman, S. \& Reid, S. (2009). Job authority and health: Unrevealing the competing suppression and explanatory influences. Social Science \& Medicine, 69(11), 1616-1624. 
Smollan, R., \& Pio, E. (2017). Organisational change, identity and coping with stress. New Zealand Journal of Employment Relations, 43(1), 56-82.

Spagnoli, P., Buono, C., Kovalchuk, L. S., Cordasco, G., \& Esposito, A. (2021). Perfectionism and burnout during the COVID-19 Crisis: A two-wave cross-lagged study. Frontiers in Psychology, 11, 4087.

Swider, B. W. \& Zimmerman, R. D. (2010). Born to burnout: a meta-analytic path model of personality, job burnout, and work outcomes. Journal of Vocational Behaviour, 76(3), 487506.

Van Heule, S., Rosseel, Y., Vlerick, P., Van de Ven, B., \& Declercq, F. (2012). The factorial validity and measurement invariance of the Utrecht Burnout Scale General version (UBOSA). Behaviour \& Organizations, 25(2), 192-201.

Walter, S. L., Seibert, S. E., Goering, D., \& O’Boyle, E. H. (2019). A tale of two sample sources: Do results from online panel data and conventional data converge? Journal of Business and Psychology, 34(4), 425-52.

Warr, P. (2005). Work, well-being, and mental health. In J. Barling, E.K. Kelloway, \& M.R. Frone (Eds.), Handbook of Work Stress (pp. 547-574). Sage Publications.

Wheeler, D. L., Vassar, M., Worley, J. A., \& Barnes, L. L. (2011). A reliability generalisation metaanalysis of coefficient alpha for the Maslach Burnout Inventory. Educational and Psychological Measurement, 71(1), 231-244.

Williams, L., Vandenberg, R., \& Edwards, J. (2009). 12 structural equation modeling in management research: A guide for improved analysis. The Academy of Management Annals, 3(1), 543-604.

Worley, J. A., Vassar, M., Wheeler, D. L., \& Barnes, L. L. (2008). Factor structure of scores from the Maslach Burnout Inventory: A review and meta-analysis of 45 exploratory and confirmatory factor-analytic studies. Educational and Psychological Measurement, 68(5), 797-823. 